\title{
Protocol
}

\section{Response of osteoarthritis biomarkers after a rehabilitation program: study protocol}

\author{
Eliane Antonioli ${ }^{1}$, Felipe B. D. Oliveira ${ }^{1}$, Rosana R. Campedelli ${ }^{1}$, Alessandro R. Zorzi ${ }^{1}$, \\ Danielli Specialli ${ }^{1}$, Sudha Agarwal $^{2}$, Mario Ferretti ${ }^{1 *}$
}

\author{
${ }^{1}$ Hospital Israelita Albert Einstein, São Paulo, Brazil \\ ${ }^{2}$ Department of Biomedical Engineering, Ohio State University, Columbus, OH, USA
}

Received: 15 October 2019

Revised: 22 November 2019

Accepted: 28 November 2019

\section{*Correspondence: \\ Dr. Mario Ferretti, \\ E-mail: ferretti@einstein.br}

Copyright: () the author(s), publisher and licensee Medip Academy. This is an open-access article distributed under the terms of the Creative Commons Attribution Non-Commercial License, which permits unrestricted non-commercial use, distribution, and reproduction in any medium, provided the original work is properly cited.

\begin{abstract}
Background: Knee osteoarthritis is a progressive degenerative joint disease and remains a leading cause of pain, physical impairment and decline in health-related quality of life in adults. Despite its incidence being amongst the highest in chronic diseases, effective biomarkers are not available to assist in its management. The main goal of this study is to identify mediators that serve as biomarkers and investigate if the levels of these biomarkers will be correlated to the efficacy of a rehabilitation program.

Methods: This is a prospective cohort study with 65 participants. Patients with mild-to-moderate symptomatic knee osteoarthritis will be recruited. The Rehabilitation Program will consist of three session/week during eight weeks. Assessment about functional evaluation will be performed before and after treatment, using the Western Ontario and McMaster Universities Osteoarthritis Index (WOMAC) and EuroQOL-5D (Euro quality of life - five dimension) scales, Visual Analog Scale (VAS), and physical function tests (time up and go, isometric strength testing and kinematic gait analysis). Serum levels of classical pro-inflammatory cytokines, hyaluronan and high mobility group box 1 protein (HMGB-1) will be evaluated. The primary outcome is the change in WOMAC scale from baseline to end. Statistical analyses will be used to determine correlation of physical improvement and serum biomarkers. Adverse events will be monitored throughout the study.

Conclusions: This trial expect to study the correlation between the anti-inflammatory effects of rehabilitation program derived factors that may be involved in suppressing cytokine induction via suppressing HMGB-1.

Trial registration: Clinicaltrials.gov - NCT02964624.
\end{abstract}

Keywords: Osteoarthritis, Biomarkers, Rehabilitation, Pain, Knee

\section{INTRODUCTION}

Osteoarthritis (OA) is the most common type of degenerative joint disease that afflicts overall $3.7-4.1 \%$ adult population globally. ${ }^{1-3}$ It is a major economic burden due to loss of work, pain, functional disability, and excessive health care costs. ${ }^{1}$ Furthermore, despite its incidence being amongst the highest in chronic diseases, effective biomarkers, diagnostic aids and imaging technology are not available to assist in the management of OA. There is no cure or disease modifying drugs available to treat the disease. Therefore, effective biomarkers and diagnostic tests are direly needed to monitor the disease progression and treatment.

The management of OA is broadly divided into nonpharmacological, pharmacological, and surgical 
treatments. Mounting evidence suggests that nonpharmacological treatment such as exercise/physical therapy may lower the risk for onset or progression of $\mathrm{OA}$ by mitigating inflammation. These therapies are effective in relieving pain, improving joint function, and may slow disease progression. However, the mechanical unloading and overloading, as seen in disuse and overuse, lead to upregulation of several proinflammatory molecules and enhance tissue degradation, whereas, dynamic moderate mechanical loading exerts antiinflammatory and anti-catabolic effects on articular cartilage by suppressing mediators of inflammation. ${ }^{4-8}$ However, the lack of robust biomarkers to measure the effectiveness of physical therapies/exercise, represent a critical gap in biotechnology, obliterating the progress in the optimal application of these therapies.

The joint breakdown in OA is driven by low-grade inflammation of cartilage, bone, and synovium. The catabolic processes associated with $\mathrm{OA}$ progression are marked by changes in proinflammatory molecules and mediators in the joint tissue and in the circulation. ${ }^{9-12}$ In human and mouse OA, changes in these molecules can be initiated by acute joint trauma or subtle changes in joint mechanics. ${ }^{13-16}$ However, diagnosis of OA is usually based on the clinical and radiographic changes, which are obtained during quite late-stage of disease and have poor sensitivity for monitoring disease progression.

Recently, high mobility group box chromosomal protein 1 (HMGB-1) has been suggested to be markedly upregulated in OA. However, presently there are no inhibitors of HMGB-1 that could be used therapeutically. We have observed that gentle exercise is the only tool that can mitigate HMGB-1 production by local and systemic macrophages, and thus inflammation. ${ }^{17}$ In this study, we would evaluate the serum concentration of HMGB-1 as a biomarker in OA patients and relate it to the functional capacity of knee joints in OA patients after rehabilitation treatment. A correlation of physical improvement after rehabilitation treatment and serum biomarkers will be performed.

Exercise and physical activity in combination with patient education has been showed efficient results as first treatments to reduce knee pain and improve physical function and consequently quality of life. ${ }^{18-21}$ Several systematic reviews described that exercise and physical activity are important interventions as well as safe in rehabilitation for older adults with knee pain improving function. $^{22-25}$ In summary, exercise therapy is considered to be an efficacious, safe and low cost treatment available to OA.

The development and following up of preventive strategies for $\mathrm{OA}$ is mainly dependent upon a better understanding of the molecular mechanisms and the identification of reliable biomarkers that reflect specific biological or pathological processes associated with OA. However, much is still unknown about the relationships of circulating levels of biomarkers of cartilage degradation and the development and progression of OA.

Products resulting from the synthesis or degradation of articular cartilage have been suggested as candidates to biomarkers of OA. For example, the biomarkers Cpropeptide of type II procollagen (CPII), hyaluronic acid (HA), and cartilage oligomeric matrix protein fragments (COMP), C-telopeptide of type II collagen (CTX-II) and type II collagen cleavage neopeptide $(\mathrm{C} 2 \mathrm{C})$ in serum and urine have all been shown to be elevated based on the presence and severity of knee OA. ${ }^{24,25}$

Considering that inflammation has been suggested as key mechanism in the pathogenesis of knee OA, cytokines, both pro- and anti-inflammatory, have been studied as possible candidates for biochemical markers in both human and animal models. ${ }^{26,27}$ Currently, the inflammatory/immunological markers were among the relatively 'best'-performing markers with regard to burden, prognostic and/or treatment efficacy, as is shown in recent reviews and study. ${ }^{25,26,28}$

Additionally, it has been shown that HMGB-1, a highly conserved protein that was previously known as a DNAbinding protein, was recently identified as a potent proinflammatory cytokine during the inflammatory process, is present in high levels in synovial fluid and tissues from patients with rheumatoid arthritis and also from rats with experimental arthritis. ${ }^{19,29-31}$ Recent study showed a correlation between synovial fluid levels of HMGB-1 and disease severity of primary knee OA, suggesting the HMGB-1 as a possible OA biomarker prognostic. ${ }^{32}$

Considering that OA is an inflammatory disease, the importance of the understanding of the effect of rehabilitation treatment in the systemic inflammatory profile is crucial for rehabilitative treatment.

Our central hypothesis is that the circulating levels of specific molecules (HMGB-1) could serve as robust biomarkers for quantitative measures of OA burden, prognosis, progression or treatment efficacy and the blood level of HGMB-1 will be decreased after the rehabilitation program and functional improvement will be expected in patients with mild OA. The main purpose is to establish novel biomarkers to monitor the OA disease activity. We will correlate serum levels of HMGB-1 and pro-inflammatory cytokines with physical improvement after rehabilitation treatment in patients with mild to moderate knee osteoarthritis. The levels of these biomarkers will be correlated to the efficacy of the rehabilitation program using a functional scale termed Western Ontario \& McMaster Universities index (WOMAC), one quality of life scale known EuroQol, the pain scale called Visual Analogue Scale (VAS). This will confirm the effectiveness of rehabilitative therapies can be monitored via assessment of circulating HMGB-1 and cytokines. We expect to find that rehabilitation program for knee osteoarthritis improves clinical function and pain 
as well as acts as an anti-inflammatory therapy by decreasing inflammatory molecules on serum of the patients. After conclusion of this study, we hope to define the HMGB-1 as a possible novel biomarker to quantitatively measure the improvement outcomes of knee osteoarthritis.

\section{METHODS}

\section{Study design and setting}

This is a prospective cohort study composed by 65 participants with mild to moderate knee osteoarthritis. The allocation ratio is based in the inclusion criteria. The design of this study is according to recommendations for interventional trials (SPIRIT) guidelines. Prior to the rehabilitation protocol, volunteers will perform one adaptation session to all the functional tests that will be applied to minimize learning bias. In the second session after 5 minutes warm up in a cycle ergometer, the Time Up and Go test will be applied, followed by the isometric strength test. In the third day, after the same warm up, one repetitions maximum testing will be conducted. At the end of the rehabilitation program, all the tests will be repeated. Blood samples will be collected at the beginning, before any tests or exercise, and at the end of the program.

The study has been approved by the local Research Ethics Committee (CAAE n ${ }^{\circ}$ 55053616.1.0000.0071). All participants will sign a consent form to agree to participate in the study. The study will last a total of 24 months. The initial 2 months will be for protocol adjustments and recruitment period. The subsequent 12 months will cover the treatment and follow-up periods (these will overlap the recruitment phase) and a final 10month period will be dedicated to data analysis.

\section{Inclusion criteria}

Women and men aged 45-65 years. Patients with clinical knee osteoarthritis according to the American College of Rheumatology Clinical Criteria. ${ }^{9}$ Patients who have Kellgren and Lawrence (KL) radiographic osteoarthritis grade 2 and 3 (mild to moderate radiographic osteoarthritis).

\section{Exclusion criteria}

Patients with severe knee osteoarthritis according to the KL classification (grade 4). Those with other known major musculoskeletal impairments in the lower extremities or the back or prostheses in any joint of the lower extremities. Patients with known serious coronary heart diseases or cancer, body mass index $>35$, those who have been scheduled for surgery in any joint. Persons who already perform sports related moderate physical activity more than two times a week. Patients with history of knee replacement surgery or high tibial osteotomy, any knee surgery or corticosteroid injections within the previous 6 months. And those patients who have missed more than two rehabilitation sessions during the program or more than two consecutive sessions.

\section{Recruitment}

The participants will be enrolled in the study after a comprehensive explanation of each step. The assessment and collection of outcomes will be performed for one experienced physical therapist and two orthopedists in, who have expertise with the questionnaires (WOMAC, EQ-5D, Timed Up and Go Test (TUG), VAS), the radiographic analysis will be carried out by the orthopedists. The biomarker assessment will be performed at Instituto de Ensino e Pesquisa (IEP) of Hospital Israelita Albert Einstein (HIAE) in Brazil coordinated for one biologist. The isometric strength testing will be executed for physical educator specialized in the isokinetic evaluation and the gait analysis will be assessed in the laboratory specialized in movement analysis of HIAE coordinated for one experienced physical therapist. The data analysis will be performed in collaboration with Ohio State University.

\section{Interventions}

The sample will be recruited from the community and a simple questionnaire will be performed for a functional evaluation in a preliminary interview for a patient selection. In the second appointment, the participants will be evaluated for two blind orthopedists according to the radiographic degree of knee osteoarthritis (KL grade <3) and will be incorporated in the study according to the inclusion criteria. Figure 1 presents the flow chart diagram of the participants in the study.

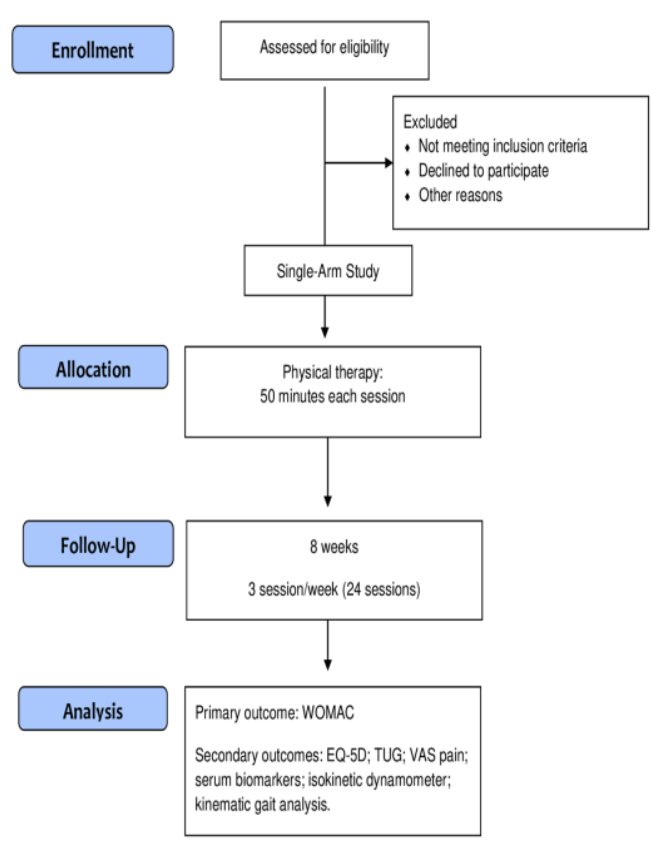

Figure 1: Participants through the study. 


\section{Rehabilitation protocol}

The rehabilitation protocol will consist of a threesession/week standard exercise program for eight weeks. Each session will begin with the evaluation of pain by the volunteer (VAS). If the pain is higher than four, an analgesic protocol (AP) containing ultra-sound, laser or TENS, will be applied. After the AP, the volunteer will just execute the rehabilitation protocol if the pain decreased to or lower than four.

Starting with a warm up exercise in a cycle ergometer (CEE), for 5 minutes at free cadence, then the intensity will be set at $90 \%$ of the intensity obtained during the incremental test and maintained for $10 \mathrm{~min}$.

Afterward, four sets (8-12 repetitions) of three different resistance exercises (RE) (leg press, knee extension and knee flexion) will be performed at $70 \%$ of the load correspondent to $1 \mathrm{RM}$ test with one-minute interval between sets and exercises. During CEE Borg scale will be taken every two minutes and during RE the OMNI scale taken at the end of every set to monitor the intensity. ${ }^{12}$

At the end of the session, cool down global stretching exercises for inferior members will be performed and the VAS evaluated again. Ten seconds of tension for each stretching position targeting the hamstrings, quadriceps, gluteus maximums, gastrocnemius, thigh adductors and abductors.

\section{Rehabilitation intensity parameters}

\section{Cycle ergometer incremental test}

After 5 minutes warm up at free cadence in a cycle ergometer, the intensity will be increased every 3 minutes until the volunteer register 11 to 13 in Borg scale which is consider a moderate intensity and to be correlated to blood lactate concentration threshold and anaerobic threshold, respectively. ${ }^{10}$

\section{One repetition maximum}

The test starts with a warm up of 5 to 10 repetitions with estimated moderate load $( \pm 40-60 \%$ of $1 \mathrm{RM})$. After 1 minute rest 3 to 5 repetitions are performed with higher load $( \pm 60-80 \%$ of $1 \mathrm{RM})$ with 5 minutes rest. Then one attempt at estimated $1 \mathrm{RM}$ is given. If the volunteer fails to lift the load for two repetitions the test stops, otherwise after 5 minutes rest the load is increased and the test is performed again. Only five attempts per day are permitted to maintain the test reliable.

\section{Primary outcome evaluation}

Change from baseline to end rehabilitation program in Western Ontario and McMaster Universities Arthritis Index (WOMAC)
Participants will answer the questionnaire WOMAC, at beginning and end of rehabilitation program, for the assessment of pain and physical function during daily activities. The WOMAC measures five items for pain (score range 0-20), two for stiffness (score range 0-8), and 17 for functional limitation (score range 0-68). For each item, the possible range of scores is therefore 0-100. Items are summed for each subscale, resulting in possible ranges as follows i.e., pain is 0-500, stiffness is 0-200, physical function is $0-1700$. Most commonly, summing the items for all three subscales creates a total WOMAC score. Higher scores on the WOMAC indicate worse pain, stiffness, and functional limitations. ${ }^{18}$

\section{Secondary outcomes}

\section{Health-related quality of life}

Health-related QOL (EuroQOL-5D, EQ-5D) will be assessed by the EQ-5D (EuroQoL Group 1990). ${ }^{19}$ Also, day 0 (pre-rehabilitation) and day 90 (after 3 months of treatment) will be evaluated.

\section{Timed Up and Go Test}

The purpose of Timed Up and Go Test (TUG) is to test basic mobility skills of frail elderly persons. The test consists of the time measurement in seconds for a person to rise from sitting from a standard arm chair, walk 3 meters, turn, walk back to the chair, and sit down. It will also be performed on day 0 (pre-rehabilitation) and day 90 (after 3 months of treatment)

\section{Visual Analog Scale}

The pain Visual Analog Scale (VAS) is a unidimensional measure of pain intensity, which has been widely used in diverse adult populations, including those with rheumatic disease. ${ }^{18}$ It will also be performed on day 0 (prerehabilitation) and day 90 (after 3 months of treatment).

\section{Biomarker assessments}

Serum samples will be obtained by venous blood collection (three vacutainer tubes, each containing $6 \mathrm{ml}$ ). The venous blood will be collected from the antecubital vein prior to initiation of therapy (pre-rehabilitation) and 24-hour post-exercise session following 3 and 6 weeks of therapy. After being centrifuged at $1800 \mathrm{~g}$ for 10 minutes, samples will be stored at -80 degrees Celsius in $1 \mathrm{ml}$ aliquots. The following kits will be:

- $\quad$ LXSAHM- Human Magnetic Luminex Assay (9 Plex, R\&D), for detection of nine molecules simultaneously: TNF-ALPHA; IL-6; IL-8/CXCL8; L-10; IL-1 BETA/IL-1F2; IL-17E/IL-25; CL3/MIP1 ALPHA; LEPTIN; IL-18.

- $\quad$ LMPM - Human Magnetic Luminex Assay (3 Plex, $\mathrm{R} \& \mathrm{D})$, for detection of three metalloproteinases: MMP-1, -3, -13 . 
Analysis using ELISA (Enzyme-linked immunosorbent assays) methodologies of molecules relate to cartilage degradation and synthesis:

\section{Collagen synthesis}

CPII (type II collagen carboxy propeptide) - (Ibex Pharmaceuticals Inc., cat. \#60-1003-001),

\section{Collagen degradation}

C2C (neoepitope created by the cleavage of type II collagen by collagenases) - (Ibex Pharmaceuticals Inc., cat. Cat. \# 60-1001-001), CS846 (epitope on chondroitin sulfate chains of the cartilage proteoglycan aggrecan) (Ibex Pharmaceuticals Inc., cat. \#60-1004), COMP (Cartilage Oligomeric Matrix Protein) (R\&D Systems, cat. DCMP0), HA (hyaluronic acid) (R\&D Systems, cat. DHYAL0); HMGB1High mobility group box-1 (CloudCorp, cat. SEA399Hu).

\section{Radiographic knee osteoarthritis progression (joint space and KL score)}

Radiological examination of conventional radiographic procedure will be performed at baseline and at the end of rehabilitation treatment.

\section{Isometric strength testing}

Isometric strength will be measured by the peak torque (PT) obtained during knee extension (PTE), knee flexion (PTF) and maximal voluntary contraction (MVC), using an isokinetic dynamometer (Biodex, Shirley, NY). During test the volunteer was seated and securely strapped the dynamometer chair. For knee extension MVC the thigh and leg angle will be $75^{\circ}\left(0^{\circ}=\right.$ total extension) and for knee flexion MVC thigh-leg angle will be $40^{\circ}\left(0^{\circ}=\right.$ total extension $)$. Two attempts of five seconds MVC will be performed for each joint movement, with 3 minutes of interval between them. The isometric PTE and PTF will be determined as the highest PT values between attempts. It will also be performed on day 0 (prerehabilitation) and day 90 (after 3 months of treatment).

\section{Kinematic gait analysis}

Acquisition of kinematic data will be performed using the Vicon MX 40 (Oxford MetricsGroup; UK) system, which consists of 10 infrared cameras with a maximum frequency of 2000 frames per second (fps), and will employ four components: emitter; medium; reflector; and receiver. The images will be processed using ULTRANET ${ }^{\circledR}$ and Nexus® software. During test participants will perform the task in a simulated way, walking in the kinematic data collection track. The task will be to wander in a demarcated track in the ground of $1.5 \times 4.0 \mathrm{~m}, 12$ times. The three-dimensional kinematic data of the joints of the pelvis, hip, knee and ankle will be processed, that is, selected walking cycles through Nexus ${ }^{\circledR}$ software, and transformed into graphs relating the angular position as a function of time, in addition data will be collected from the Linear kinematics such as, gait speed and cadence.

Participants underwent three-dimensional gait analysis while walking barefoot and at a self-selected speed. Reflective markers were positioned over lower limb anatomical landmarks during walking as well as over the medial femoral epicondyles and medial malleoli during an initial static standing trial used to determine joint centre locations. Kinematic data were collected using 8 high-speed digital video cameras (Motion Analysis Corp., Santa Rosa, CA) sampling at $120 \mathrm{~Hz}$. Kinetic data were sampled at $1200 \mathrm{~Hz}$ using 2 floor-mounted force platforms (Advanced Mechanical Technology Inc., Watertown, MA) positioned in the middle of the walkway and synchronized with the cameras. Net joint moments were calculated using commercially available software (Orthotrak, Motion Analysis Corp.).

\section{Sample size}

The estimation of sample size was determined on the basis of a greater improvement of subscale physical function of the WOMAC score, using G. Power 3.15 software. Based on $10 \%$ expected difference between baseline measure and after rehabilitation treatment and a standard deviation of 30 on physical function of WOMAC, 65 patients are needed with significance level of 0.05 , and power of $80 \%$.

\section{Statistical analysis}

For continuous variables, normal distribution will be evaluated with the Kolmogorov-Smirnov test. Results of normally distributed continuous variables will be expressed as the mean value $\pm \mathrm{SD}$, and those for nonnormally distributed continuous variables will be expressed as the median (interquartile range). Categorical variables will be summarized as frequencies or percentages. Differences between the baseline and end of rehabilitation treatment will be analyzed using unpaired ttest or Chi-square test when appropriate. Differences between before and after treatment will be analyzed by one-way analysis of variance (ANOVA) or the KruskalWallis analysis followed by Tukey 'post hoc' analysis.

As the HMGB-1 levels is not normally distributed, logarithmic (log) transformed values will be performed for multiple comparisons among the time analyzed. Spearman rank correlation coefficient will be employed to determine the correlation between HMGB-1 levels and WOMAC score.

Multinomial logistic regression analysis will be performed to assess the independent predictors of WOMAC scores. As the HMGB-1 levels were not normally distributed, log transformations values will be also performed for multinomial logistic regression. All statistical analyses will be performed using SAS Statistical Software Package (SAS Institute, 2001) A 
value of $\mathrm{p}<0.05$ (two-tailed) will be considered statistically significant.

\section{Data management and monitoring}

In this research, a data monitoring committee (DMC) will be not necessarily due to the short duration of the study and low risks involved for the participants. The institutional review boards (IRBs) also have responsibility for monitoring the safety of trial participants at the institutions. The data monitoring will be a liability of the three main investigators according to the institution. The access of the interim results will be a duty of the main and second investigators that includes the decision to terminate the trial in any stage of the study.

The harms will be evaluated during and after the tests for the rehabilitative program, biomarker assessment, isokinetic strength assessment and gait analysis. Any adverse event will be reported to the researchers involved and communicated to the main investigator according to the IRBs description.

\section{DISCUSSION}

The results from this study expect to validate whether rehabilitation program is able to act as anti-inflammatory signal in knee OA patients, by monitoring serum HMGB1 and pro-inflammatory cytokines levels. Also, to correlate the modulation in serum levels of HMGB-1 with clinical improvement of knee joint in OA patients after rehabilitation program.

The primary aims of this study will improve the understanding of the mechanisms of actions of rehabilitation program in inflammatory molecules. And, possibility will help in the development of specific methods to treat joints afflicted with OA by using levels of HMGB-1 and cytokines as tools to assess the progression and resolution of the disease.

There is a lack of studies about the correlation of biomarkers with the clinical and functional results of rehabilitation programs. Loeser et al performed a clinical trial to evaluate the effect of dietary weight loss with and without exercise on biomarkers in adults with KOA. ${ }^{33,34}$ Four biochemical markers of inflammation were used: CRPM, C1M, C2M and C3M. Weight loss with or without exercise, but not exercise alone, were correlated with $\mathrm{C} 1 \mathrm{M}$ and $\mathrm{C} 3 \mathrm{M}$ decrease. None of the markers measured were associated with the change in WOMAC pain, but C3M and CRPM were weakly associated with WOMAC function. Mobasheri et al published an extensive review about biomarkers in OA. They do not report any trial about correlation of biomarkers and rehabilitation programs. According to them, the biomarker-related studies published include some of the usual suspects such as COMP, HA, ADAMTS-4, the aggrecan ARGS neo-epitope and the type II collagen markers CTX-II, C2C and COLL2-1 NO2.

Recent studies suggest that other cytokines may exert pro- inflammatory effects on articular cartilage in synovial joints. Experiments for the identification of a specific molecule, HMGB-1, is novel and not yet studied in the context of OA treatment. The HMGB-1 has been suggested to have a significant role in cartilage inflammation and tissue destruction observed in OA.

\section{CONCLUSION}

This trial expects to study the correlation between the anti-inflammatory effects of rehabilitation program derived factors that may be involved in suppressing cytokine induction via suppressing HMGB-1.

Funding: São Paulo Research Foundation supports this work in collaboration with Ohio State University (FAPESP/OSU, number 2015/50274-5)

Conflict of interest: None declared

Ethical approval: The study was approved by the IRB of Hospital Israelita Albert Einstein (CAAE $n^{\circ}$ 55053616.1.0000.0071).

\section{REFERENCES}

1. Weinstein SL, Jacobs JJ, Goldberg MJ. Osteoarthritis of the knee. $\mathrm{N}$ Engl $\mathrm{J}$ Med. 2006;354(23):2508-9.

2. Kapoor M, Martel-Pelletier J, Lajeunesse D, Pelletier JP, Fahmi H. Role of proinflammatory cytokines in the pathophysiology of osteoarthritis. Nat Rev Rheumatol. 2011;7(1):33-42.

3. Cross M, Smith E, Hoy D, Nolte S, Ackerman I, Fransen M, et al. The global burden of hip and knee osteoarthritis: estimates from the global burden of disease 2010 study. Ann Rheum Dis. 2014;73(7):1323-30.

4. McAlindon TE, Bannuru RR, Sullivan MC, Arden NK, Berenbaum F, Bierma-Zeinstra SM, et al. OARSI guidelines for the non-surgical management of knee osteoarthritis. Osteoarthrit Cartilage. 2014;22(3):363-88.

5. Zhang W, Moskowitz RW, Nuki G, Abramson S, Altman RD, Arden N, et al. OARSI recommendations for the management of hip and knee osteoarthritis, Part II: OARSI evidence-based, expert consensus guidelines. Osteoarthrit Cartilage. 2008;16(2):137-62.

6. Henrotin Y, Raman R, Richette P, Bard H, Jerosch $\mathrm{J}$, Conrozier $\mathrm{T}$, et al. Consensus statement on viscosupplementation with hyaluronic acid for the management of osteoarthritis. Semin Arthritis Rheum. 2015;45(2):140-9.

7. Strand V, McIntyre LF, Beach WR, Miller LE, Block JE. Safety and efficacy of US-approved viscosupplements for knee osteoarthritis: a 
systematic review and meta-analysis of randomized, saline-controlled trials. J Pain Res. 2015;8:217-28.

8. Campbell KA, Erickson BJ, Saltzman BM, Mascarenhas R, Bach BRJ, Cole BJ, et al. Is Local Viscosupplementation Injection Clinically Superior to Other Therapies in the Treatment of Osteoarthritis of the Knee: A Systematic Review of Overlapping Meta-analyses. YJARS. 2015;31(10):2036-45.e14.

9. Altman R, Asch E, Bloch D, Bole G, Borenstein D, Brandt $\mathrm{K}$, et al. Development of criteria for the classification and reporting of osteoarthritis. Classification of osteoarthritis of the knee. Diagnostic and Therapeutic Criteria Committee of the American Rheumatism Association. Arthritis Rheum. 1986;29(8):1039-49.

10. 10. Hsueh M-F, Onnerfjord P, Kraus VB. Biomarkers and proteomic analysis of osteoarthritis. Matrix Biol. 2014;39:56-66.

11. Kurtz S, Ong K, Lau E, Mowat F, Halpern M. Projections of primary and revision hip and knee arthroplasty in the United States from 2005 to 2030. J Bone Joint Surg Am. 2007;89(4):780-5.

12. Kraus VB, Blanco FJ, Englund M, Henrotin Y, Lohmander LS, Losina E, et al. OARSI Clinical Trials Recommendations: Soluble biomarker assessments in clinical trials in osteoarthritis. Osteoarthrit Cartilage. 2015;23(5):686-97.

13. Kotlarz H, Gunnarsson CL, Fang H, Rizzo JA. Insurer and out-of-pocket costs of osteoarthritis in the US: evidence from national survey data. Arthritis Rheum. 2009;60(12):3546-53.

14. Little CB, Hunter DJ. Post-traumatic osteoarthritis: from mouse models to clinical trials. Nat Rev Rheumatol. 2013;9(8):485-97.

15. Saúde, Osteoartrose 2012. Available at: http://www.brasil.gov.br/saude/2012/04/osteoartros. Accessed on 9 June 2015.

16. Christiansen BA, Guilak F, Lockwood KA, Olson SA, Pitsillides AA, Sandell LJ, et al. Non-invasive mouse models of post-traumatic osteoarthritis. Osteo Cart. 2015;23(10):1627-38.

17. Nam JP, Blazek A, Ferretti, M. Exercise suppresses HMGB1 expression and functions in activated macrophages. In submission; 2017.

18. Hochberg MC, Altman RD, April KT, Benkhalti M, Guyatt G, McGowan J, et al. American College of Rheumatology 2012 recommendations for the use of nonpharmacologic and pharmacologic therapies in osteoarthritis of the hand, hip, and knee. Arthritis Care Res (Hoboken). 2012;64(4):465-74.

19. Scaffidi P, Misteli T, Bianchi ME. Release of chromatin protein HMGB1 by necrotic cells triggers inflammation. Nature. 2002;418(6894):191-5.

20. Terada C, Yoshida A, Nasu Y, Mori S, Tomono Y, Tanaka M, et al. Gene expression and localization of high-mobility group box chromosomal protein-1 (HMGB-1) in human osteoarthritic cartilage. Acta Med Okayama. 2011;65(6):369-77.
21. Hunt MA, Pollock CL, Kraus VB, Saxne T, Peters $\mathrm{S}$, Huebner JL, et al. Relationships amongst osteoarthritis biomarkers, dynamic knee joint load, and exercise: results from a randomized controlled pilot study. BMC Musculoskel Dis. 2013;14:115.

22. Quicke JG, Foster NE, Thomas MJ, Holden MA. Is long-term physical activity safe for older adults with knee pain? a systematic review. Osteoarthrit Cartilage. 2015;23(9):1445-56.

23. Kyostio-Moore S, Nambiar B, Hutto E, Ewing PJ, Piraino S, Berthelette $P$, et al. STR/ort mice, a model for spontaneous osteoarthritis, exhibit elevated levels of both local and systemic inflammatory markers. Comp Med. 2011;61(4):346-55.

24. van Spil WE, DeGroot J, Lems WF, Oostveen JCM, Lafeber FPJG. Serum and urinary biochemical markers for knee and hip-osteoarthritis: a systematic review applying the consensus BIPED criteria. Osteoarthrit Cartilage. 2010;18(5):605-12.

25. Lafeber FPJG, van Spil WE. Osteoarthritis year 2013 in review: biomarkers; reflecting before moving forward, one step at a time. Osteoarthrit Cartilage. 2013;21(10):1452-64.

26. Mabey T, Honsawek S. Cytokines as biochemical markers for knee osteoarthritis. World J Orthop. 2015;6(1):95-105.

27. Jayadev C, Rout R, Price A, Hulley P, Mahoney D. Hyaluronidase treatment of synovial fluid to improve assay precision for biomarker research using multiplex immunoassay platforms. J Immunol Methods. 2012;386(1-2):22-30.

28. Imamura M, Ezquerro F, Marcon Alfieri F, Vilas Boas L, Tozetto-Mendoza TR, Chen J, et al. Serum levels of proinflammatory cytokines in painful knee osteoarthritis and sensitization. Int $\mathrm{J}$ Inflam. 2015;2015:329792.

29. Lotze MT, Tracey KJ. High-mobility group box 1 protein (HMGB1): nuclear weapon in the immune arsenal. Nat Rev Immunol. 2005 Apr;5(4):331-42.

30. Kokkola R, Sundberg E, Ulfgren A, Palmblad K, Li $\mathrm{J}$, Wang $\mathrm{H}$, et al. High mobility group box chromosomal protein 1: A novel proinflammatory mediator in synovitis. Arthritis Rheum. 2002;46:2598-603.

31. Taniguchi N, Kawahara K-I, Yone K, Hashiguchi T, Yamakuchi M, Goto M, et al. High mobility group box chromosomal protein 1 plays a role in the pathogenesis of rheumatoid arthritis as a novel cytokine. Arthritis Rheum. 2003;48(4):971-81.

32. Li Z-C, Cheng G-Q, Hu K-Z, Li M-Q, Zang W-P, Dong $Y-Q$, et al. Correlation of synovial fluid HMGB-1 levels with radiographic severity of knee osteoarthritis. Clin Invest Med. 2011;34(5):E298.

33. Loeser RF, Beavers DP, Bay-Jensen AC, Karsdal MA, Nicklas BJ, Guermazi A, et al. Effects of dietary weight loss with and without exercise on interstitial matrix turnover and tissue inflammation biomarkers in adults with knee osteoarthritis: the 
Intensive Diet and Exercise for Arthritis trial (IDEA). Osteoarthrit Cartilage. 2017;25(11):1822-8.

34. Mobasheri A, Bay-Jensen AC, van Spil WE, Larkin J, Levesque MC. Osteoarthritis Year in Review 2016: biomarkers (biochemical markers). Osteoarthrit Cartilage. 2017;25(2):199-208.
Cite this article as: Antonioli E, Oliveira FBD, Campedelli RR, Zorzi AR, Specialli D, Agarwal S, et al. Response of osteoarthritis biomarkers after a rehabilitation program: study protocol. Int J Clin Trials 2020;7(1):35-42. 